\title{
A Multicenter Phase II Study of Gemcitabine plus S-1 Chemotherapy for Advanced Biliary Tract Cancer
}

\author{
SHIHO ARIMA ${ }^{1}$, KYOKO SHIMIZU $^{2}$, TOMOYOSHI OKAMOTO ${ }^{3}$, MASAO TOKI $^{4}$, \\ YUTAKA SUZUKI $^{5}$, NAOHIRO OKANO ${ }^{1}$, DAISUKE NARUGE ${ }^{1}$, KIRIO KAWAI $^{1}$, \\ TAKAAKI KOBAYASHI, AKIYOSHI KASUGA ${ }^{1}$, HIROSHI KITAMURA ${ }^{1}$, \\ ATUKO TAKASU $^{1}$, FUMIO NAGASHIMA ${ }^{1}$, MASANORI SUGIYAMA ${ }^{5}$ and JUNJI FURUSE ${ }^{1}$ \\ ${ }^{1}$ Department of Medical Oncology, Kyorin University Faculty of Medicine, Tokyo, Japan; \\ ${ }^{2}$ Institute of Gastroenterology, Tokyo Women's Medical University, Tokyo, Japan; \\ ${ }^{3}$ Department of Surgery, The Jikei University Daisan Hospital, Tokyo, Japan; \\ ${ }^{4}$ Third Department of Internal Medicine, Kyorin University Faculty of Medicine, Tokyo, Japan; \\ ${ }^{5}$ Department of Surgery, Kyorin University Faculty of Medicine, Tokyo, Japan
}

\begin{abstract}
Background: Gemcitabine (GEM) plus cisplatin $(C D D P)$ chemotherapy has been used worldwide as the standard first-line treatment for advanced biliary tract cancer (BTC). A phase II trial has also suggested promising activity of GEM plus $S$-1 chemotherapy against advanced BTC. The aim of this study was to evaluate the efficacy and safety of GEM plus $S-1$ chemotherapy in patients with advanced BTC. Patients and Methods: The eligibility criteria were as follows: histologically-proven BTC, unresectable or recurrent disease, ECOG performance status (PS) 0-1 regardless of previous treatment. Gemcitabine was administered intravenously at the dose of $1,000 \mathrm{mg} / \mathrm{m}^{2}$ over 30 min on days 1 and 8 , and $S-1$ was administered orally at doses of 60/80/100 mg/day based on the BSA, from day 1 to day 14 , every 3 weeks. The primary endpoint was the response rate according to RECIST, ver. 1.1 , and the secondary endpoints were the frequency/severity of toxicities, progression-free survival (PFS) and overall survival (OS). Results: A total of 38 patients were enrolled between August 2008 and November 2011. There were 19 men and 19 women, with a median age of 66 years (range $=44-81$ years). Seven patients had a previous history of first-line or adjuvant chemotherapy after surgery. The PS was 0 and 1 in 30 and 7 patients, respectively. The treatment response was classified as partial response in 6 patients (15.8\%) and as
\end{abstract}

Correspondence to: Professor Junji Furuse, Department of Medical Oncology, Kyorin University School of Medicine, 6-20-2, Shinkawa, Mitaka-shi Tokyo, 181-8611, Japan. Tel: +81 422475511, Fax: +81 422440604, e-mail: jfuruse@ks.kyorin-u.ac.jp

Key Words: Biliary tract cancer, chemotherapy, gemcitabine, S-1, phase II study. stable disease in 18 patients (47.4\%). The median PFS and OS were 5.8 and 15.9 months, respectively. The toxicity was generally mild, and the most common grade 3/4 toxicities were leukopenia (31.6\%), neutropenia (36.8\%), nausea/vomiting (2.6\%), and diarrhea (2.6\%). There was one treatment-related death due to interstitial pneumonia. Conclusion: Our study revealed that gemcitabine plus $S-1$ chemotherapy was well-tolerated and exhibited favorable antitumor activity in patients with advanced BTC.

Biliary tract cancer comprises extrahepatic cholangiocarcinoma (EHCC), gallbladder cancer (GBC) and ampulla of Vater cancer (AVC); intrahepatic cholangiocarcinoma (IHCC) is also often included in clinical trials of treatments for biliary tract cancer. The incidence is high in Asia and south America and approximately 25,000 patients are annually diagnosed as having biliary tract cancer in Japan (1). While BTC is recognized as a relatively rare disease in Western countries, the incidence of intrahepatic cholangiocarcinoma is gradually increasing in Western countries (2-6).

Biliary tract cancer is a common cause of cancer-related death in Asia, including Japan, with an estimated 18,000 deaths occurring from this cancer annually in Japan. While surgery currently remains the only potentially curative treatment, many patients are diagnosed at an advanced, unresectable stage of the disease. Even among patients with advanced disease who are treated by curative surgery, disease recurrence occurs at a high frequency (7). Thus, although systemic chemotherapy plays an important role in the treatment of advanced biliary tract cancer, the prognosis of this cancer remains extremely poor.

In the ABC-02 trial, a randomized phase III study carried out in the U.K. to compare gemcitabine alone with gemcitabine plus cisplatin (GC) in patients with unresectable 
or recurrent biliary tract cancer, a statistically significantly higher overall survival rate was obtained in the GC arm as compared to the gemcitabine-alone arm (8). In the BT22 study, a randomized controlled trial carried out to confirm the efficacy/safety of GC therapy as compared to that of gemcitabine monotherapy in Japanese patients, similar results to those of the ABC-02 study were obtained (9). Thus, GC therapy is now recognized as the global standard for chemotherapy of unresectable biliary tract cancer.

On the other hand, S-1 is an oral anticancer drug that consists of a mixture of tegafur (FT), 5-chloro-2,4dihydroxypyridine (CDHP), and potassium oxonate (Oxo); FT is a prodrug of 5-FU, CDHP is a competitive inhibitor of dihydropyrimidine dehydrogenase (DPD), an enzyme that catalyzes the degradation of 5-FU, and oxo is a competitive inhibitor of orotate phosphoribosyltransferase (OPRT) and inhibits the phosphorylation of 5-FU in the gastrointestinal tract, reducing the serious gastrointestinal toxicity of 5-FU. A phase II study of S-1 for advanced biliary tract cancer demonstrated promising activity, with a response rate of $35 \%$, median time-to-progression of 3.7 months, and median overall survival of 9.4 months (10). Based on these results, S-1 was approved for the treatment of biliary tract cancer in Japan in 2007.

Furthermore, combination of gemcitabine and S-1 has also been demonstrated to show promising activity against biliary tract cancer and pancreatic cancer (11-13). Therefore, we conducted this multicenter phase II study to evaluate the efficacy and safety of gemcitabine plus S-1 chemotherapy (GS therapy) in patients with unresectable biliary tract cancer.

\section{Patients and Methods}

Patient eligibility. The eligibility criteria of patients for enrollment in the phase II study were: histologically or cytologically confirmed adenocarcinoma or adenosquamous carcinoma; clinical diagnosis of biliary tract cancer (IHCC, EHCC, GBC or AVC); measurable disease on computed tomography $(\mathrm{CT})$ or magnetic resonance imaging (MRI) unresectable disease; age $\geq 20$ years old; Eastern Cooperative Oncology Group (ECOG) performance status (PS) of 0-1; capable of sufficient oral intake; preserved organ functions (leukocyte count $3,500 / \mathrm{mm}^{3}$ or more, neutrophil count $1,500 / \mathrm{mm}^{3}$ or more, platelet count $100,000 / \mathrm{mm}^{3}$ or more, hemoglobin level $9 \mathrm{~g} / 100 \mathrm{~mL}$ or more, serum creatinine concentration $1.2 \mathrm{mg} / \mathrm{dL}$ or less, serum total bilirubin level $2 \mathrm{mg} / \mathrm{dL}$ or less $(3 \mathrm{mg} / \mathrm{dL}$ or less in patients with biliary drainage) and serum aspartate transaminase (AST) and alanine transaminase (ALT) levels $150 \mathrm{IU} / \mathrm{L}$ or less; life expectancy $\geq 8$ weeks; willing to provide written informed consent. The exclusion criteria were: serious complications such as active infection, active gastrointestinal ulcer, cardiac disease, and/or renal disease; central nervous system metastasis; massive pleural effusion or ascites; symptomatic interstitial pneumonitis; pregnant or lactating women. In this study, patients with a previous history of chemotherapy were not excluded, because we expected that GS therapy would be efficacious as a second-line treatment as well. This study was
Table I. Patient characteristics $(n=38)$.

\begin{tabular}{lc}
\hline Characteristics & \\
\hline Male & $19(50 \%)$ \\
Female & $19(50 \%)$ \\
Age (years) & \\
$\quad$ Median age (range) & $66(44-81)$ \\
ECOG performance status & $31(82 \%)$ \\
0 & $6(16 \%)$ \\
1 & $1(2 \%)$ \\
2 & $12(37 \%)$ \\
Location of the primary tumor & $14(31 \%)$ \\
Intrahepatic bile duct & $9(24 \%)$ \\
Extrahepatic bile duct & $3(8 \%)$ \\
Gallbladder & $7(18 \%)$ \\
Ampulla of Vater & $6(5: 1)$ \\
Prior chemotherapy & 1 \\
Adjuvant chemotherapy (S-1: GEM) & \\
First-line chemotherapy (S-1) & \\
\hline
\end{tabular}

ECOG, Eastern Cooperative Oncology Group.

conducted with the approval of the local institutional review boards of all the participating centers (approval number: 20-2).

Treatment methods. The dose and dosing schedule of GS therapy were as follows; $1000 \mathrm{mg} / \mathrm{m}^{2}$ of gemcitabine was administered by intravenous infusion on days 1 and 8 , and $\mathrm{S}-1$ at $30 \mathrm{mg} / \mathrm{m}^{2}$ $\left(60 \mathrm{mg} /\right.$ day for a body surface area [BSA] $<1.25 \mathrm{~m}^{2}, 80 \mathrm{mg} /$ day for $1.25 \leq$ BSA $<1.50 \mathrm{~m}^{2}$, and $100 \mathrm{mg} /$ day for BSA $\geq 1.50 \mathrm{~m}^{2}$ ) was administered orally twice daily from days 1 to 14 , repeated every 3 weeks. The treatment cycles were repeated until documentation of disease progression, emergence of unacceptable toxicity or patient refusal to continue the treatment. Prophylactic administration of antiemetic agents such as dexamethasone and/or a 5-HT3 receptor antagonist was allowed at the investigator's discretion.

If a patient's leukocyte count was $<3,000 / \mathrm{mm}^{3}$, platelet count was $<100,000 / \mathrm{mm}^{3}$, total bilirubin level was $>3.0 \mathrm{mg} / \mathrm{dL}$, AST/ALT level was $>150 \mathrm{IU} / \mathrm{L}$, or serum creatinine level was $>1.4 \mathrm{mg} / \mathrm{dL}$, initiation of the next treatment cycle was postponed until recovery of these data. If, during a treatment cycle, a patient showed a leukocyte count of $<2,000 / \mathrm{mm}^{3}$, platelet count of $<70,000 / \mathrm{mm}^{3}$, diarrhea $>$ grade 1 , mucositis in the oral cavity $>$ grade 2 , or rash $>$ grade 2 , gemcitabine was not given on day 8 and S-1 administration was suspended. In patients who developed grade 4 leukopenia or neutropenia, febrile neutropenia or infection with grade 3 leukopenia or neutropenia, grade 4 thrombocytopenia or grade 3 thrombocytopenia requiring transfusion, or grade 3 rash, the dose of gemcitabine was reduced to $800 \mathrm{mg} / \mathrm{m}^{2}$. In patients who showed grade 4 leukopenia or neutropenia, febrile neutropenia or infection with grade 3 leukopenia or neutropenia, grade 4 thrombocytopenia or grade 3 thrombocytopenia requiring transfusion, a serum creatinine level $\geq 1.5 \mathrm{mg} / \mathrm{dL}$, diarrhea $\geq$ grade 3 , mucositis in the oral cavity $\geq$ grade 3 , or rash $\geq$ grade 3 , the dose of $\mathrm{S}-1$ was reduced by $20 \mathrm{mg} /$ day in the subsequent cycle.

Assessment of response and toxicity. Physical examination, complete blood cell counts, serum chemistries and urinalysis were 
Table II. Tumor response.

\begin{tabular}{|c|c|c|c|c|c|}
\hline & All patients $(n=38)$ & IHCC patients $(n=13)$ & EHCC patients $(n=12)$ & GBC patients $(n=10)$ & AVC patients $(n=3)$ \\
\hline Complete response & $1(2.6 \%)$ & 0 & $1(8.3 \%)$ & 0 & 0 \\
\hline Partial response & $5(13.2 \%)$ & 0 & $1(8.3 \%)$ & $4(40 \%)$ & 0 \\
\hline Stable disease & $20(52.6 \%)$ & $9(69.2 \%)$ & $6(50.0 \%)$ & $4(40 \%)$ & $1(33.3 \%)$ \\
\hline Progressive disease & $6(15.8 \%)$ & $3(23.1 \%)$ & $2(16.7 \%)$ & 0 & $1(33.3 \%)$ \\
\hline Not evaluable & $6(15.8 \%)$ & $1(7.7 \%)$ & $2(16.7 \%)$ & $2(20 \%)$ & $1(33.3 \%)$ \\
\hline Objective response rate & $15.8 \%$ & 0 & $16.7 \%$ & $40.0 \%$ & 0 \\
\hline Disease control rate & $68.4 \%$ & $69.2 \%$ & $66.7 \%$ & $40.0 \%$ & $33.3 \%$ \\
\hline
\end{tabular}

IHCC, Intrahepatic cholangiocarcinoma; EHCC, extrahepatic cholangiocarcinoma; GBC, gallbladder cancer; AVC, ampulla of Vater cancer.

performed at the baseline and at least twice every 3 weeks after the start of treatment. Dynamic CT or MRI was performed to evaluate the response at 4- to 6-week intervals after the start of treatment. Computed tomography or MRI was performed by obtaining contiguous transverse sections using the helical scanning method, at a section thickness of $5 \mathrm{~mm}$. Tumor response was assessed using the Response Evaluation Criteria in Solid Tumors. Objective responses were confirmed by a second evaluation performed at least 4 weeks later. Toxicity was graded according to the National Cancer Institute Common Terminology Criteria for Adverse Events, version 3.0.

Study design. The primary endpoint of this study was the overall response rate and the secondary endpoints were the frequency/severity of adverse events, OS, and PFS. The threshold response rate was defined as $17.5 \%$, and the expected response rate was set as $35 \%$. A sample size of 40 would ensure that there was $80 \%$ statistical power at a one-sided significance level of $5 \%$. The accrual period was set at 2 years and the follow-up period at 1 year.

Statistical analysis. Progression-free survival was calculated from the first day of treatment until the appearance of evidence of tumor progression, clinical progression, or death due to any cause. Overall survival was calculated from the first day of treatment until death due to any cause. Survival data were analyzed using the KaplanMeier method. The tumor response, treatment toxicity, and survival were evaluated based on intention-to-treat analyses.

\section{Results}

Patient characteristics. A total of 40 patients were enrolled between August 2008 and November 2011. Two patients were excluded because of rapid clinical deterioration before the first administration of the anticancer chemotherapy. The patient characteristics are listed in Table I. There were 19 men and 19 women, with a median age of 66 years (range $=44-81$ years). The PS was 0 and 1 in 30 and 7 patients, respectively. Seven patients had a previous history of receiving first-line or adjuvant chemotherapy after surgery. One patient had received S- 1 treatment as first-line chemotherapy. Of the six patients who had received prior adjuvant chemotherapy, five patients received S-1 treatment and one patient received gemcitabine alone.
Treatment. A total of 257 cycles of GS therapy were administered, with a median of 5 cycles per patient (range, 1 to 27 cycles). Two patients were excluded because of rapid clinical deterioration before the first administration of anticancer treatment. In all the 38 enrolled patients, gemcitabine was administered at $100 \%$ of the initially planned dose and $\mathrm{S}-1$ was administered at the dose of $80 \mathrm{mg} / \mathrm{m}^{2}$ in 10 patients and at the dose of $100 \mathrm{mg} / \mathrm{m}^{2}$ in 24 patients.

Of the 38 patients in whom the treatment was discontinued, twenty-six $(68.4 \%)$ showed disease progression and $2(2.6 \%)$ refused further continuation of the treatment because of adverse events such as gastric ulcer and mucositis. Treatment was discontinued because of adverse events in six (15.8\%) patients, including interstitial pneumonia in three patients, one with ileus diarrhea and one with anorexia. One treatment-related death due to interstitial pneumonia occurred. Two patients were lost to follow-up because of moving to other hospitals or moving from their houses. In one patient, the treatment was discontinued based on the attending physician's advice after patient was confirmed to show a complete pathological response.

After the GS treatment was discontinued, twenty-two patients received second-line treatment, including systemic chemotherapy with fixed dose-rate infusion of gemcitabine with oral fluorinated pyrimidine compound S-1 (FGS therapy) in six patients, gemcitabine in four patients, $S-1$ in three patients, gemcitabine plus oxaliplatin (GEMOX therapy) in three patients and a clinical trial treatment in one patient; three patients underwent surgery. The remaining two patients received only best supportive care.

Efficacy. One patient showed complete response (CR), while five patients showed partial responses (PRs), representing an overall objective response rate of $15.8 \%$ (Table II). Stable disease was observed in twenty patients (62.5\%), with an overall disease control rate of $68.4 \%$. Of the seven patients with a previous history of having received chemotherapy, one showed PR, two showed SD, two showed PD and disease was not evaluated in 1 patient. The median overall survival time was 15.9 months (95\% CI=8.9-23.0 months; 


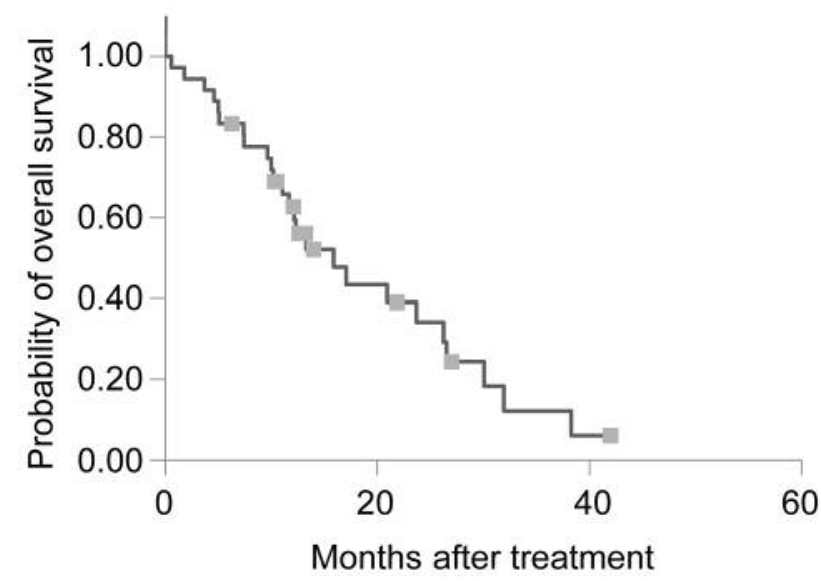

Figure 1. Overall survival in 38 patients administered GS therapy. The median overall survival period was 15.9 months.

Figure 1) and the median time-to-progression was 5.8 months (95\% CI, 3.1-8.5 months; Figure 2).

Safety. Table III shows the treatment-related adverse events. Grade 3 or 4 toxicities were observed in 15 of the 40 patients (38\%). The major toxicities in Grade 3 or 4 patients were leukopenia $(31.6 \%, 12 / 38)$, neutropenia $(36.8 \%, 14 / 38)$, anemia $(2.6 \%, 1 / 38)$, ALT elevation $(2.6 \%, 1 / 38)$, AST elevation $(2.6 \%, 1 / 38)$, nausea/vomiting $(2.6 \%, 1 / 38)$, diarrhea $(2.6 \%, 1 / 38)$ and interstitial pneumonia $(2.6 \%$, 1/38). The reported serious adverse events were grade 3 diarrhea and ileus, in addition to one case of treatmentrelated death caused by interstitial pneumonia. There were no cases of febrile neutropenia.

\section{Discussion}

This multicenter phase II study was conducted to evaluate preliminary the efficacy and safety of GS therapy in patients with unresectable biliary tract cancer, not only as first-line, but also as second-line treatment. The primary endpoint was the objective response rate, with the threshold response rate defined as $17.5 \%$ and expected response rate set at $35 \%$ in this study. The overall response rate was $15.8 \%$, therefore, the primary endpoint was not met in this study. We speculate the following as some of the reasons for the endpoint not having been met; some patients had a previous history of treatments and in some patients, the response could not be evaluated as they moved to another hospital. However, the median PFS (5.8 months) and OS (15.9 months) were promising, being comparable to those reported from other studies of GS therapy $(12,14)$.

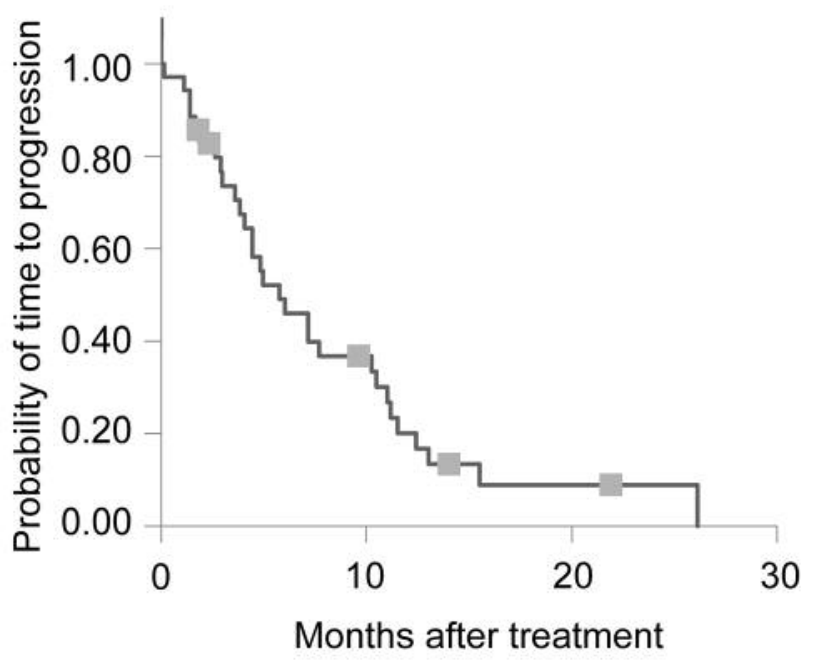

Figure 2. Progression-free survival in 38 patients treated with GS therapy. The median progression-free survival period was 5.8 months.

Table III. Treatment-related adverse events $(n=38)$ : worst grade reported during the treatment period.

\begin{tabular}{|c|c|c|c|c|c|}
\hline & \multicolumn{4}{|c|}{ Grade (n) } & \multirow{2}{*}{$\begin{array}{c}\text { Grade } 3 / 4 \\
\mathrm{n}(\%)\end{array}$} \\
\hline & 1 & 2 & 3 & 4 & \\
\hline \multicolumn{6}{|l|}{ Hematological } \\
\hline Leukopenia & 8 & 10 & 11 & 1 & $12(31.6)$ \\
\hline Neutropenia & 4 & 5 & 11 & 3 & $14(36.8)$ \\
\hline Anemia & 17 & 7 & 1 & 0 & $1(2.6)$ \\
\hline Thrombocytopenia & 15 & 6 & 0 & 0 & 0 \\
\hline T-Bil elevation & 3 & 2 & 0 & 0 & 0 \\
\hline ALT elevation & 12 & 1 & 1 & 0 & $1(2.6)$ \\
\hline AST elevation & 17 & 0 & 1 & 0 & $1(2.6)$ \\
\hline Hypoalbuminemia & 18 & 7 & 0 & 0 & 0 \\
\hline \multicolumn{6}{|l|}{ Non-hematological } \\
\hline Mucositis & 6 & 3 & 0 & 0 & 0 \\
\hline Anorexia & 8 & 4 & 0 & 0 & 0 \\
\hline Nausea/vomiting & 7 & 3 & 1 & 0 & $1(2.6)$ \\
\hline Diarrhea & 4 & 3 & 1 & 0 & $1(2.6)$ \\
\hline Interstitial pneumonia & 2 & 0 & 1 & 0 & $1(2.6)$ \\
\hline Dyspnea & 1 & 0 & 0 & 0 & 0 \\
\hline Abdominal pain & 1 & 0 & 0 & 0 & 0 \\
\hline Rash & 5 & 3 & 0 & 0 & 0 \\
\hline Fever & 1 & 0 & 0 & 0 & 0 \\
\hline Alopecia & 7 & 0 & 0 & 0 & 0 \\
\hline Malaise & 7 & 1 & 0 & 0 & 0 \\
\hline Duodenal ulcer & 0 & 1 & 0 & 0 & 0 \\
\hline Pigmentation & 6 & 0 & 0 & 0 & 0 \\
\hline
\end{tabular}

GS therapy has been reported to be generally welltolerated in previous clinical trials for biliary tract cancer and pancreatic cancer. In the current study, the most common 
toxicities were neutropenia and leukopenia; grade $3 / 4$ neutropenia and leukopenia developed in $36.8 \%$ and 31.6 of patients, respectively. However, there was no patients with febrile neutropenia. As for non-hematological toxicities, grade $3 \mathrm{AST}$ and ALT elevation was observed in one patient $(2.6 \%)$, and grade 3 nausea/vomiting, diarrhea, and interstitial pneumonitis were observed in one patient $(2.6 \%)$. There was one treatment-related death due to interstitial pneumonia. These safety profiles are comparable with those reported from a previous randomized phase II study conducted to compare the safety/efficacy of GS therapy and S-1 alone (JCOG0805); in this aforementioned study, however, febrile neutropenia was observed in $20 \%$ of cases and there were two treatment-related deaths caused by interstitial pneumonitis and myocardial infarction, respectively. Interstitial pneumonitis was also observed in the current study, therefore, close attention should be paid for the development of early symptoms such as dry cough, dyspnea and persistent fever.

Biliary tract cancer is generally recognized as a relatively rare disease compared to other gastrointestinal cancers, such as gastric and colorectal cancers. Although GC therapy is currently recognized as the standard treatment for patients with unresectable biliary tract cancer based on results of the ABC-02 trial, few large clinical trials have been conducted for patients with biliary tract cancer. In Japan, S-1 is approved for the treatment of biliary tract cancer, based on the results of a small phase II study of S-1 alone $(15,16)$, and it is necessary to conduct further clinical trials to confirm the efficacy and safety. A randomized phase II study (JCOG0805) was conducted to compare the efficacy/safety of GS therapy with that of S-1 monotherapy and GS therapy was found to show more promising activity compared to S-1 alone; the response rates were $36.4 \%$ in the GS arm and $17.4 \%$ in the S-1 arm, and the median OS durations were 12.5 months in the GS arm and 9.0 months in $\mathrm{S}-1 \mathrm{arm}$. As a result, GS therapy is now considered as a valid treatment alternative for biliary tract cancer to GC therapy, because GS therapy is associated with a lower incidence of gastrointestinal toxicities such as nausea/vomiting and anorexia than GC therapy and the venous infusion time is much shorter in GS therapy than in GC therapy. Therefore, a non-inferiority phase III trial between GC therapy and GS therapy (JCOG1113) is currently ongoing (UMIN000010667). Furthermore, a triplet regimen consisting of gemcitabine, a platinum agent and a fluoropyrimidine has also been investigated. A phase II study of gemcitabine plus cisplatin plus fluorouracil, which was a relatively small study conducted in 21 patients, showed a response rate of $33.3 \%$ and median OS of 18.8 months (17). A phase II study of gemcitabine plus cisplatin plus S-1 (GCS therapy) also showed promising results, with a response rate of $24 \%$ and median OS of 16.2 months (18).
As a consequence, a phase III trial comparing GCS therapy with GC therapy is currently ongoing (UMIN000014371).

Although GC therapy is established as the first-line treatment for biliary tract cancer, most patients develop disease progression. In order to improve the survival, effective second-line chemotherapy needs to be developed. The impact of second-line therapy after gemcitabine-based chemotherapy was assessed in the ABC-02 and BT-22 studies (19). In the ABC-02 trial conducted in the U.K., only $17 \%$ of the patients received further chemotherapy, mostly 5-FU based chemotherapy. On the other hand, more than $70 \%$ of the patients received post-study chemotherapy in the BT-22 study conducted in Japan; more than $60 \%$ of the patients were treated with $\mathrm{S}-1$ as the second-line therapy. In spite of the difference in the rate of application of secondline therapy, the overall survival in the two studies was quite similar. Furthermore, the phase II study of S-1 as failed to demonstrate promising activity of the drug as second-line treatment, with a response rate of $7.5 \%$, median PFS of 2.5 months, and median OS of 6.8 months (20). So far, therefore, the impact of second-line therapy remains unclear in patients with gemcitabine-refractory biliary tract cancer. In this study, we found that GS therapy may be indicated in patients with a previous history of chemotherapy. In this study, seven patients who had a previous history of chemotherapy were included; even among these patients, one showed PR, and two showed SD.

This study had several limitations. First of all, only a small number of patients were included. The purpose of this study is to preliminarily explore the efficacy and safety of GS therapy for unresectable biliary tract cancer. Therefore, we evaluated the response rate and safety profile in a small number of patients. Secondly, this study included not only patients without a previous history of chemotherapy, but also those with a previous history of chemotherapy. It was designed to evaluate the efficacy of GS therapy as secondline treatment. However, it seemed to be difficult to accurately evaluate the response rate.

In conclusion, although the current phase II study of GS therapy failed to meet the primary endpoint (that is, an objective response rate of $17.5 \%$ ), it demonstrated promising activity for improving the survival in patients with unresectable biliary tract cancer. GS therapy may also be effective as second-line treatment after GC therapy.

\section{References}

1 Foundation for Promotion of Cancer Research. Cancer statistics in Japan-2015. http://ganjoho.jp/data/reg_stat/statistics/brochure/ 2015/cancer_statistics_2015_fig_J.pdf

2 Yao KJ, Jabbour S, Parekh N, Lin Y and Moss RA: Increasing mortality in the United States from cholangiocarcinoma: an analysis of the National Center for Health Statistics Database. BMC Gastroenterology 16(1): 117-125, 2016. 
3 Altekruse SF, Petrick JL, Rolin AI, Cuccinelli JE, Zou Z, Tatalovich $Z$ and McGlynn KA: Geographic variation of intrahepatic cholangiocarcinoma, extrahepatic cholangiocarcinoma, and hepatocellular carcinoma in the United States. PLoS One 10(3): e0120574, 2015

4 Patel T: Increasing incidence and mortality of primary intrahepatic cholangiocarcinoma in the United States. Hepatology 33(6): 1353-1357, 2001.

5 Njei B: Changing pattern of epidemiology in intrahepatic cholangiocarcinoma. Hepatology 60(3): 1107-1178, 2014.

6 Saha SK, Zhu AX, Fuchs CS and Brooks GA: Forty-year trends in cholangiocarcinoma incidence in the U.S.: intrahepatic disease on the rise. Oncologist 21(5): 594-599, 2016.

7 Furuse J, Takada T, Miyazaki M, Miyakawa S, Tsukada K, Nagino M, Kondo S, Saito H, Tsuyuguchi T, Hirata K, Kimura F, Yoshitomi H, Nozawa S, Yoshida M, Wada K, Amano H and Miura F: Guidelines for chemotherapy of biliary tract and ampullary carcinomas. J Hepatobiliary Pancreat Surg 15(1): 5562, 2008.

8 Valle J, Wasan H, Palmer DH, Cunningham D, Anthoney A, Maraveyas A, Madhusudan S, Iveson T, Hughes S, Pereira SP, Roughton $\mathrm{M}$ and Bridgewater $\mathrm{J}$ : $\mathrm{ABC}-02$ Trial Investigators: Cisplatin plus gemcitabine versus gemcitabine for biliary tract cancer. N Engl J Med 362(14): 1273-1281, 2010.

9 Okusaka T, Nakachi K, Fukutomi A, Mizuno N, Ohkawa S, Funakoshi A, Nagino M, Kondo S, Nagaoka S, Funai J, Koshiji M, Nambu Y, Furuse J, Miyazaki M and Nimura Y: Gemcitabine alone or in combination with cisplatin in patients with biliary tract cancer: a comparative multicentre study in Japan. Br J Cancer 103(4): 469-474, 2010.

10 Furuse J, Okusaka T, Boku N, Ohkawa S, Sawaki A, Masumoto $\mathrm{T}$ and Funakoshi A: S-1 monotherapy as first-line treatment in patients with advanced biliary tract cancer: a multicenter phase II study. Cancer Chemother Pharmacol 62(5): 849-855, 2008.

11 Ueno H, Okusaka T, Furuse J, Yamao K, Funakoshi A, Boku N, Ohkawa S, Yokosuka O, Tanaka K, Moriyasu F, Nakamori S and Sato T: Multicenter phase II study of gemcitabine and S-1 combination therapy (GS Therapy) in patients with metastatic pancreatic cancer. Jpn J Clin Oncol 41(8): 953-958, 2011.

12 Sasaki T, Isayama $\mathrm{H}$, Nakai $\mathrm{Y}$, Ito $\mathrm{Y}$, Kogure $\mathrm{H}$, Togawa $\mathrm{O}$, Toda N, Yasuda I, Hasebe O, Maetani I, Sasahira N, Hirano K, Tsujino T, Tada $M$ and Omata M: Multicenter, phase II study of gemcitabine and S-1 combination chemotherapy in patients with advanced biliary tract cancer. Cancer Chemother Pharmacol 65(6): 1101-1107, 2010.
13 Kim HS, Kim HY, Zang DY, Oh HS, Jeon JY, Cho JW, Park CK, Kim JH, Kim MJ, Ha HI, Kim JH, Han B, Song H, Kwon JH, Choi DR and Jung JY: Phase II study of gemcitabine and S-1 combination chemotherapy in patients with metastatic biliary tract cancer. Cancer Chemother Pharmacol 75(4): 711-718, 2015.

14 Morizane C, Okusaka T, Mizusawa J, Takashima A, Ueno M, Ikeda M, Hamamoto $\mathrm{Y}$, Ishii $\mathrm{H}$, Boku $\mathrm{N}$ and Furuse $\mathrm{J}$ : Randomized phase II study of gemcitabine plus S-1 versus S-1 in advanced biliary tract cancer: a Japan Clinical Oncology Group trial (JCOG 0805). Cancer Sci 104(9): 1211-1216, 2013.

15 Ueno H, Okusaka T, Ikeda M, Takezako Y and Morizane C: Phase II study of S-1 in patients with advanced biliary tract cancer. Br J Cancer 91(10): 1769-1774, 2004.

16 Furuse J, Okusaka T, Boku N, Ohkawa S, Sawaki A, Masumoto $\mathrm{T}$ and Funakoshi A: S-1 monotherapy as first-line treatment in patients with advanced biliary tract cancer: a multicenter phase II study. Cancer Chemother Pharmacol 62(5): 849-855, 2008.

17 Kanai M, Hatano E, Kobayashi S, Fujiwara Y, Marubashi S, Miyamoto A, Shiomi H, Kubo S, Ikuta S, Yanagimoto H, Terajima H, Ikoma H, Sakai D, Kodama Y, Seo S, Morita S, Ajiki T, Nagano H and Ioka T: A multi-institution phase II study of gemcitabine/cisplatin/S-1 (GCS) combination chemotherapy for patients with advanced biliary tract cancer (KHBO 1002). Cancer Chemother Pharmacol 75(2): 293-300, 2015.

18 Yamashita Y, Taketomi A, Itoh S, Harimoto N, Tsujita E, Sugimachi K, Gion $\mathrm{T}$ and Maehara Y: Phase II trial of gemcitabine combined with 5-fluorouracil and cisplatin (GFP) chemotherapy in patients with advanced biliary tree cancers. Jpn J Clin Oncol 40(1): 24-28, 2010.

19 Furuse J, Okusaka T, Bridgewater J, Taketsuna M, Wasan H, Koshiji $\mathrm{M}$ and Valle J: Lessons from the comparison of two randomized clinical trials using gemcitabine and cisplatin for advanced biliary tract cancer. Crit Rev Oncol Hematol 80(1): 3139, 2011.

20 Suzuki E, Ikeda M, Okusaka T, Nakamori S, Ohkawa S, Nagakawa T, Boku N, Yanagimoto H, Sato T and Furuse J: A multicenter phase II study of S-1 for gemcitabine-refractory biliary tract cancer. Cancer Chemother Pharmacol 71(5): 1141-1146, 2013.

Received December 7, 2016 Revised January 26, 2017 Accepted January 27, 2017 